\title{
Ultrasound assessment of gastric emptying in premature infants treated with non-invasive ventilatory support
}

\author{
Dalibor Kurepa ${ }^{1}$, Richard Schanler ${ }^{1}$, Barry Weinberger ${ }^{1}$, and Arkar Ye Hlaing ${ }^{1}$ \\ ${ }^{1}$ Cohen Children's Medical Center Division of Neonatology
}

November 26, 2020

\begin{abstract}
Abstract Background: Nasal CPAP introduces positive pressure of air into both trachea and stomach, which may affect gastric emptying. The rate of gastric emptying can be estimated by US by two validated techniques: "antral cross-sectional area" (2-dimensional estimate of the surface area at the gastric antrum), and "spheroid gastric volume" (3-dimensional estimate of the stomach content volume). No study examined gastric emptying rate in infants on bubble CPAP (bCPAP). Objective: To compare gastric emptying rates in neonates on machine-derived nasal CPAP (MD-nCPAP) with those on bCPAP. Methods: Ultrasound measurements of the amount of milk in the stomach were performed before feeding and at 1, 2, and 3 hours after the start of feeding, using both the ACSA and spheroid methods. Rates of gastric emptying were calculated during the "early" (1-2 hours) and "late" (2-3 hours) phases after feeding. Results: We recruited 32 infants (25-34 weeks gestational age). Seventeen infants were treated with MD-nCPAP [median birth weight $1015 \mathrm{~g}$ (IQR: 870 to 1300), gestational age 28 weeks (IQR: 27 to 29), postnatal age 20 days (IQR: 14 to 28)], while 15 infants were treated with bCPAP [median birth weight $960 \mathrm{~g}$ (IQR: 855 to 1070), gestational age 27 weeks (IQR: 26 to 28), postnatal age 17 days (IQR: 15 to 25 )]. Gastric emptying rates (\% emptied/min) were significantly faster in the "early" compared to the "late" phase for all infants. There were no significant differences in the rates of gastric emptying (either "early" or "late") or volumes of gastric residuals between infants receiving MD-nCPAP or bCPAP, measured by either method. Conclusions: Gastric emptying is faster during the "early" compared to the "late" phase. Gastric emptying rates are not different in infants receiving MD-nCPAP vs bCPAP.
\end{abstract}

\section{Ultrasound assessment of gastric emptying in premature infants treated with non-invasive ventilatory support}

Arkar Ye Hlaing, Barry Weinberger, Richard Schanler, Dalibor Kurepa

Affiliations: Department of Pediatrics, Division of Neonatal-Perinatal Medicine, Cohen Children's Medical Center, New Hyde Park, New York

Corresponding author: Dalibor Kurepa, MD

Division of Neonatal-Perinatal Medicine, Cohen Children's Medical Center

269-01 76th Avenue, Suite CH-344, New Hyde Park, NY 11040

Tel: 718-470-3440

Email: dkurepa@northwell.edu

Short Title: Gastric emptying in neonates on CPAP

Keywords: POC ultrasound, gastric emptying, preterm, CPAP, bubble CPAP, ACSA

Abbreviations: continuous positive airway pressure (CPAP), gastrointestinal tract (GIT), respiratory distress syndrome (RDS), machine-derived nasal CPAP (MD-nCPAP), bubble CPAP (bCPAP), orogastric tube 
(OGT), total parenteral nutrition (TPN), antral cross-sectional area (ACSA), ultrasound (US), necrotizing enterocolitis (NEC), very low birth weight (VLBW), gestational age (GA), corrected gestational age (CGA)

\section{1 | INTRODUCTION}

Nasal continuous positive airway pressure (CPAP) and surfactant replacement have been recommended as the first line treatment for premature infants with respiratory distress syndrome (RDS) [1]. CPAP is the most studied non-invasive respiratory support modality in premature infants. It delivers continuous distending pressure to the airways and alveoli to maintain functional residual capacity [2]. However, CPAP may exert other physiologic effects by introducing positive pressure into the gastrointestinal tract (GIT).

Optimal nutrition is critically important for premature infants, but their gastrointestinal tract motility may be impaired relative to older infants and children [3]. Jaile et al. described benign gaseous distention of the GIT in infants treated with CPAP and devised the term 'CPAP belly' [4]. Another study reported improved gastric emptying in infants on machine-derived nasal CPAP (MD-nCPAP) compared to healthy room air controls [5]. However, gastric emptying in preterm infants on other modes of non-invasive respiratory support, including widely-used bubble CPAP (bCPAP), has not been studied. Delayed gastric emptying and large gastric residual volumes have been associated with feeding intolerance and necrotizing enterocolitis (NEC) $[6,7]$.

Our primary objective was to compare ultrasound (US)-estimated gastric emptying rates in premature infants receiving full enteral feedings who are treated with either MD-nCPAP or bubble CPAP (bCPAP). The secondary objective was to determine the relationship between clinically assessed feeding tolerance and US-estimated volumes of residual stomach contents prior to feeding.

\section{2| METHODS}

We prospectively studied gastric emptying in very low birth weight (VLBW) infants treated with either MDnCPAP or bCPAP from May 2018 to March 2020. The study was approved by Northwell Health Institutional Review Board (Manhasset, NY). Informed consent was obtained from parents before enrollment. Inclusion criteria included infants 25-34 weeks of gestational age (GA) at birth receiving full enteral feedings and treated with either MD-nCPAP or bCPAP for RDS. Infants with major congenital anomalies, gastrointestinal disease or surgery, and those treated with systemic antibiotics or GIT motility medications were excluded from the study.

Per NICU protocol, infants who are not mature enough to nipple feed are fed by bolus every 3 hours via orogastric tube (OGT). After minimal enteral trophic feedings are tolerated for 1-2 days, the feedings are increased daily by $\sim 20 \mathrm{~mL} / \mathrm{kg} /$ day until full enteral feeding volumes of $160 \mathrm{ml} / \mathrm{kg} /$ day are achieved. Feedings are fortified to $24 \mathrm{kcal} / \mathrm{oz}$ using Similac Human Milk Liquid Fortifier (Abbott Nutrition, Abbott Park, IL, USA) when enteral feeding volumes reach $\sim 60 \mathrm{ml} / \mathrm{kg} /$ day. Parenteral nutrition (PN) support is discontinued when infants tolerate $\sim 120 \mathrm{~mL} / \mathrm{kg} /$ day of enteral feeding. Gavage feedings were provided over a feeding pump for 60 minutes period. The stomach is decompressed by opening the OGT to straight drainage 30 minutes after completion of feeding. All infants are examined every 3 hours for vomiting, abdominal distention, bowel sounds, bowel movement and abdominal wall softness. Gastric residuals or aspirates are checked only when an OGT is placed or replaced, or if there is a clinical indication. Feeding intolerance was defined as the withholding of feedings by the attending physician because of one or more of the following: bilious emesis, significant abdominal distention, abnormal bowel auscultation or abnormal vital signs.

Treatment of apnea of prematurity and RDS includes administration of IV or PO caffeine together with either MD-nCPAP or bCPAP. During the study period, our NICU was transitioning from using MD-nCPAP to bCPAP, with the choice dependent solely on the availability of bCPAP at the time of patient's admission. MD-nCPAP was delivered by AVEA ${ }^{\mathrm{TM}}$ (Vyaire Medical, Inc., Chicago, IL, USA) mechanical ventilator and administered through RAM cannula ${ }^{\circledR}$ (Neotech, Valencia, CA, USA). Bubble CPAP was delivered by a commercially available system (Fisher \& Paykel Healthcare Ltd., Auckland, New Zealand) connected to Babi.Plus ${ }^{\circledR}$ nasal prongs (Respiralogics, NV, USA). Oxygen saturations were maintained between $88 \%$ and 
95\% as per NICU protocol. Upper airways and mouth were gently suctioned on an as-needed basis to keep them clear. Infants' necks were kept in slight extension.

\section{1 | Imaging techniques}

Gastric emptying was assessed using 2 methods at a time when infants were receiving at least $120 \mathrm{~mL} / \mathrm{kg} /$ day of enteral feedings and no intravenous fluids. The first method is to calculate the maximum antral crosssectional area (ACSA) by measuring the longest anteroposterior and transverse diameters of the gastric antrum by real-time US (Zonare, Mindray, Shenzhen, China) [8]. The second method is to calculate the spheroid gastric volume by measuring the largest anteroposterior, longitudinal and transverse diameters of the stomach filled with milk with the US transducer positioned on the lower left lateral chest and upper abdomen $[9,10]$. Each infant had a total of 4 measurement points: before feeding ( 0 hour $)$, at 1 hour, 2 hours and 3 hours after the initiation of the feeding (Figure 1 ). Infants were kept in a flat supine position during the study period. Based on the consecutive measurements, ACSAs and spheroid volumes were used to calculate percent change in ACSA and percent change in spheroid gastric volume between two consecutive feedings as proxies for percentage of milk emptied per minute. Early gastric emptying was defined as the change in volume determination from 1 hour to 2 hours after the initiation of the feeding. Late gastric emptying defined as the change in volume from 2 hours to 3 hours after the initiation of the feeding. Values obtained by both US methods were compared between the two modes of CPAP.

Gastric residual volume percentage was calculated by dividing the gastric volume at 1 hour (immediately prior to the next feeding) by the gastric volume at 1 hour (after completion of the study feeding). Value obtained was expressed as a percentage.

A lung ultrasound score (LUS) was also determined at the 1-hour measurement point in order to ensure that the CPAP groups did not differ in the severity of their respiratory disease. The LUS score is assigned based on observations reflecting the efficacy of aeration at 6 different lung fields [12]. A recent meta-analysis has validated it for assessing the severity of RDS with high sensitivity and specificity [11].

Ultrasound gel was prewarmed and transducer sanitized before each exam. During the exam, infants were comforted with a pacifier. If an infant had oxygen desaturation below $80 \%$ or bradycardia below 80 bpm, the exam was stopped to allow the infant to recover. No sedation was used during the US exams.

\section{$2.2 \mid$ Data analysis}

Statistical analysis was carried out using SAS version 9.4 software (Cary, NC). Continuous variables were reported as medians and interquartile ranges. Categorical variables were reported as frequencies and percentages. For Table 1 and Table 2, Wilcoxon rank sum test was used to test differences in distribution of continuous variables between the two modes of ventilation. Chi-square test and Fisher's exact test were used for the comparison of proportions of categorical variables between the two modes. $\mathrm{P}$ values of $<0.05$ were considered statistically significant.

A multilevel linear mixed model was used to test for association between gastric emptying rates and ventilation modes (MD-nCPAP, bCPAP) accounting for emptying period (early, late) and gestational age $(25+0$ to $27+6$ weeks, $28+0$ to $34+6$ weeks). All possible interactions, as well as main effects, were included. Multivariable linear regression with backward elimination was used to test for association between total gastric emptying rates and ventilation mode accounting for GA. The interaction between ventilation mode and GA group, as well as the main effects were included in the model. Manual backward elimination was used to identify main effects and interactions that were statistically significant. In the case of a significant interaction term, post-hoc paired comparisons were performed, and a Bonferroni adjustment was used to account for multiple comparisons.

\section{3. | RESULTS}

We prospectively studied 32 infants: 17 receiving MD-nCPAP and 15 receiving bCPAP. Patient demographic and clinical characteristics are summarized in Table 1 and Table 2. There were no significant differences 
between the MD-nCPAP and bCPAP groups in any characteristic except corrected GA (median corrected GA in MD-nCPAP $=32$ (IQR: 31 to 33), in bCPAP $=30$ (IQR: 29 to 31), $P=0.006$ ). Lung US Score (LUS), PEEP and FiO2 were not different between the groups, suggesting that the degrees of pulmonary impairment were similar.

Gastric emptying rates calculated as percentage emptied per minute (\%emptied/min) from the values obtained by both ACSA and spheroid US methods at different time intervals were compared between MD-nCPAP and bCPAP groups. There were no significant differences in the rates measured by either ACSA or spheroid methods and between the MD-nCPAP and bCPAP groups, either as the emptying rate across the entire feeding duration or as individual early and late emptying rates (Figure 2a, 2b). The ACSA method demonstrated significantly faster gastric emptying in the early phase compared to the late phase in the $25+0$ to $27+6$ weeks GA group (Bonferroni adjusted p-value $=0.0008$ ) but not in the $28+0$ to $34+6$ weeks GA group (Bonferroni adjusted p-value $=1$ ), irrespective of ventilation group after accounting for multiple testing among paired comparisons (Figure 2a) . The spheroid method rate demonstrated significantly faster emptying in the early phase than in the late phase $(\mathrm{p}<0.0001)$ for all infants, irrespective of ventilation group and GA group (Figure 2b).

Residual gastric contents prior to the next feeding were calculated to be $42.4 \%$ in the MD-nCPAP group and $31.9 \%$ in bCPAP group when using ACSA measurements, and $23.8 \%$ and $25.8 \%$ respectively when using spheroid method. Comparison of these residual gastric contents showed no significant difference between the

MD-nCPAP and bCPAP groups by either ACSA (Figure 3a) or spheroid method (Figure 3b). None of the studied patients had emesis or measurable regurgitation.

\section{4 | DISCUSSION}

The use of CPAP to treat RDS in premature infants has been associated with a lower incidence of chronic lung disease than invasive mechanical ventilation [13]. Therefore, there has been a notable shift to noninvasive respiratory support for these infants, and the term "less may be more" has been fully embraced in neonatology. However, increased use of CPAP requires a closer examination of its potential side-effects. The presence of an oro- or nasogastric tube in many of these infants may allow the delivered and swallowed gas to move to the stomach and further down the GIT. Abdominal distention may restrict diaphragmatic movement and lung expansion, and serious complications such as intestinal perforation and necrotizing enterocolitis have been reported $[14,15,16]$. Even though we analyzed the incidence of NEC and intestinal perforation, along with BPD, and found no significant difference between MD-nCPAP and bCPAP groups, our study was not powered to detect these differences ( Table 2).

Studies using scintigraphy to determine the rate of gastric emptying in healthy term infants have yielded inconsistent results. While some studies have reported faster gastric emptying in older infants, a large recent study found that infants less than 3 months of age have faster gastric emptying than older infants and children $[17,18,19]$. As expected, gastric emptying is twice as fast in infants fed breast milk compared to formula [20]. Conversely, delayed gastric emptying has been associated with gastro-esophageal reflux in older children [21]. Using scintigraphy in VLBW infants, Gounaris et al. reported faster gastric emptying in those treated with MD-nCPAP compared to healthy controls [5]. However, the utility of scintigraphy is limited in children and infants because of the need to limit radiation exposure. US provides a noninvasive method to estimate the gastric emptying rate and can be performed at the patient's bedside $[22,23]$.

We found that neither the ACSA nor spheroid method gastric emptying rates were statistically different between infants treated with MD-nCPAP and bCPAP, analyzed by multivariable analysis adjusting for emptying phase (early, late) and GA (25-28 weeks, 28-34 weeks). The caloric density of the feedings, type of milk (expressed human milk only, donor human milk only, formula only, or mixed) or volume of milk were not significantly associated with the ACSA or spheroid rates. Corrected gestational age (CGA) was significantly different between the MD-nCPAP and bCPAP infants, but regression analysis of ACSA or spheroid rates and CGA showed no correlation between the two variables. Our findings are consistent with a recent meta-analysis that demonstrated no correlation between postmenstrual age and gastric emptying 
time [24].

A significantly faster gastric emptying was noted in the early phase (1-2 hours from the initiation of a feed) compared to the late phase (2-3 hours after initiation of a feed) when using the spheroid method, but this was seen only in the $25+0$ to $27+6$ week GA group using the ACSA method. This is consistent with previous studies showing faster gastric half-emptying times in the immediate post-prandial period using different methods of measurement [5,8,19]. A meta-analysis of 66 studies in various age groups performed by Bonner et al. revealed a non-linear emptying rate with faster early phase, especially for liquid-fed subjects compared to those fed solid food [24]. This can be explained by a high intragastric volume in the early phase, which stimulates the mucosal stretch receptors $[29,30,31,32]$.

We noted high gastric residual percentages using both methods - 41\% (30.3-49.8) by ACSA and $24.5 \%$ (16.8-32.3) by spheroid. Higher gastric residual percent measured by the ACSA method compared to the spheroid method is likely due to the antrum being the last part of the stomach to empty. It appears that traditional measurements of pre-feeding residual volumes by gastric aspiration underestimate the proportion of retained feeding by 19-25\%. This may be dependent on patient position, feeding tube size, position of the tip of the tube, milk viscosity and aspiration technique $[25,26,28]$. Our subjects at the time of the US were receiving only non-invasive respiratory support and tolerating full enteral feeding without clinical evidence of feeding intolerance. This finding brings into question the reliability and value of using aspirated gastric residuals to assess feeding intolerance or gastrointestinal pathology in neonates $[27,28]$.

\section{5 | LIMITATIONS}

One weakness of this pilot study is its small sample size. Also, it was not possible to mask the type of CPAP to investigators performing US. Nevertheless, the likelihood of bias was minimized because data on CPAP and other clinical variables were not available to the investigators who analyzed the US images and performed the gastric volume calculations. Also, gastric emptying rates were calculated based on measurements during one feeding period, which may not be representative of all feeding periods. Still, pre-feeding residual measurements for two consecutive feedings (at the beginning and end of the US period) were similar, supporting the idea that gastric emptying rates are stable.

\section{6 | CONCLUSION}

Gastric emptying rates are not different in infants receiving MD-nCPAP vs bCPAP and are faster during the "early" compared to "late" phase after feeding. The measured gastric emptying rates in all the infants appeared clinically adequate, since they all tolerated feeding. The identification of large gastric residuals by US in these stable preterm infants suggests that traditional gastric aspiration may overstate the occurrence of feeding intolerance. Nevertheless, feeding intolerance is common in VLBW infants, so larger US studies comparing modes of non-invasive and invasive ventilatory support are needed.

\section{AUTHORS CONTRIBUTIONS}

Dr. Hlaing and Dr. Kurepa contributed to study conception and design, ultrasound measurements, data interpretation, drafting and critical revision of the manuscript as well as the final approval of the manuscript. Dr. Weinberger and Dr. Schanler contributed substantially to data analysis and interpretation, drafting of the manuscript, and approval of the final manuscript.

\section{REFERENCES}

1. Committee on Fetus and Newborn, American Academy of Pediatrics. Respiratory support in preterm infants at birth. Pediatrics . 2014; 133(1):171-174.

2. Courtney SE, Barrington KJ. Continuous positive airway pressure and noninvasive ventilation. Clin Perinatol . 2007;34:73-92.

3. Bozzetti V, De Angelis C, Tagliabue PE. Nutritional approach to preterm infants on noninvasive ventilation: An update.Nutrition . 2017;37:14-17. doi: 10.1016/j.nut.2016.12.010. 
4. Jaile JC, Levin T, Wung JT, Abramson SJ, Ruzal-Shapiro C, Berdon WE. Benign gaseous distension of the bowel in premature infants treated with nasal continuous airway pressure: a study of contributing factors. AJR Am J Roentgenol . 1992;158:125-127.

5. Gounaris A, Costalos C, Varchalama L, Kokori P, Kolovou E, Alexiou N. Gastric emptying in very-lowbirth-weight infants treated with nasal continuous positive airway pressure. J Pediatr.2004;145(4):508510.

6. Cobb BA, Carlo WA, Ambalavanan N. Gastric residuals and their relationship to necrotizing enterocolitis in very low birth weight infants. Pediatrics . 2004;113(1 Pt 1):50-53. doi:10.1542/peds.113.1.50.

7. Bertino E, Giuliani F, Prandi G, Coscia A, Martano C, Fabris C. Necrotizing enterocolitis: risk factor analysis and role of gastric residuals in very low birth weight infants. J Pediatr Gastroenterol Nutr . 2009;48(4):437-442. doi:10.1097/mpg.0b013e31817b6dbe.

8. Newell S, Chapman S, Booth I. Ultrasonic assessment of gastric emptying in the preterm infant. Arch Dis Child . 1993;69:32-6.

9. Perrella SL, Hepworth AR, Simmer KN, et al. Validation of ultrasound methods to monitor gastric volume changes in preterm infants. J Pediatr Gastroenterol Nutr . 2013;57:741-9.

10. Perrella SL, Hepworth AR, Simmer KN, et al. Repeatability of gastric volume measurements and intragastric content using ultrasound in preterm infants. J Pediatr Gastroenterol Nutr . 2014;59(2):25463.

11. Wu J, Wang Y, Zhao A, Wang Z. Lung Ultrasound for the Diagnosis of Neonatal Respiratory Distress Syndrome, Ultrasound Quarterly: June 2020 - Volume 36 - Issue 2 - p 102-110 doi: 10.1097/RUQ.0000000000000490.

12. Brat R, Yousef N, Klifa R, Reynaud S, Aguilera SS, De Luca D. Lung ultrasonography score to evaluate oxygenation and surfactant need in neonates treated with continuous positive airway pressure. JAMA Pediatr . 2015;169(8):e151797.

13. Fischer HS, Buhrer C. Avoiding endotracheal ventilation to prevent bronchopulmonary dysplasia: a meta-analysis. Pediatrics . 2013;132(5):e1351-e1360.

14. Han VK, Beverley DW, Clarson C, et al. Randomized controlled trial of very early continuous distending pressure in the management of preterm infants. Early Hum Dev . 1987;15(1):21-32. doi:10.1016/0378-3782(87)90097-1.

15. Gupta S, Sinha SK, Tin W, Donn SM. A randomized controlled trial of post extubation bubble CPAP or infant flow driver CPAP in preterm infants with respiratory distress syndrome. $J$ Pediatr . 2009;154:645-50.

16. Garland JS, Nelson DB, Rice T, Neu J. Increased risk of gastrointestinal perforations in neonates mechanically ventilated with either face mask or nasal prongs. Pediatrics . 1985;76:406-10.

17. Gelfand MJ, Wagner GG. Gastric emptying in infants and children: limited utility of 1-hour measurement. Radiology . 1991;178(2):379-381.

18. Seibert JJ, Byrne WJ, Euler AR. Gastric emptying in children: unusual patterns detected by scintigraphy. AJR Am J Roentgenol . 1983;141(1):49-51. doi:10.2214/ajr.141.1.49.

19. Kwatra NS, Shalaby-Rana E, Andrich MP, et al. Gastric emptying of milk in infants and children up to 5 years of age: normative data and influencing factors. Pediatr Radiol . 2020;50(5):689-697. doi:10.1007/s00247-020-04614-3.

20. Ewer AK, Durbin GM, Morgan ME, Booth IW. Gastric emptying in preterm infants. Arch Dis Child Fetal Neonatal Ed. 1994;71(1):F24-7.

21. Lorenzo CD, Piepsz A, Ham H, Cadranel S. Gastric Emptying With Gastro-Oesophageal Reflux. Arch Dis Child. 1987;62(5):449-53.

22. Gomes H, Hornoy P, Liehn JC. Ultrasonography and gastric emptying in children: validation of a sonographic method and determination of physiological and pathological patterns. Pediatr Radiol . 2003;33(8):522-529. doi:10.1007/s00247-003-0954-1.

23. Holt S, Cervantes J, Wilkinson AA, Wallace JH. Measurement of Gastric Emptying Rate in Humans by Real-Time Ultrasound. Gastroenterology . 1986;90(4):918-923.

24. Bonner JJ, Vajjah P, Abduljalil K, et al. Does age affect gastric emptying time? A model-based meta- 
analysis of data from premature neonates through to adults. Biopharm Drug Dispos . 2015;36(4):245257.

25. Gonzales I, Duryea EJ, Vasquez E, Geraghty N. Effect of enteral feeding temperature on feeding tolerance in preterm infants. Neonatal Netw . 1995;14(3):39-43.

26. Geraldo V, Pyati S, Joseph T. et al. Gastric Residual (GR): Reliability of the Measurement. 888. Pediatr Res.1997;41:p.150A.

27. Parker L, Torrazza RM, Li Y, Talaga E, Shuster J, Neu J. Aspiration and evaluation of gastric residuals in the neonatal intensive care unit: state of the science. J Perinat Neonatal Nurs . 2015;29(1):51-E2.

28. Li YF, Lin HC, Torrazza RM, Parker L, Talaga E, Neu J. Gastric residual evaluation in preterm neonates: a useful monitoring technique or a hindrance? Pediatr Neonatol . 2014;55(5):335-340.

29. Minami H, McCallum RW. The physiology and pathophysiology of gastric emptying in humans. Gastroenterology . 1984;86(6):1592-1610.

30. Strunz UT, Grossman MI. Effect of intragastric pressure on gastric emptying and secretion. Am $J$ Physiol . 1978;235(5):E552-E555. doi:10.1152/ajpendo.1978.235.5.E552.

31. Leiper JB. Fate of ingested fluids: factors affecting gastric emptying and intestinal absorption of beverages in humans. Nutr Rev . 2015;73 Suppl 2:57-72. doi:10.1093/nutrit/nuv032.

32. Noakes TD, Rehrer NJ, Maughan RJ. The importance of volume in regulating gastric emptying. Med Sci Sports Exerc. 1991;23(3):307-313.

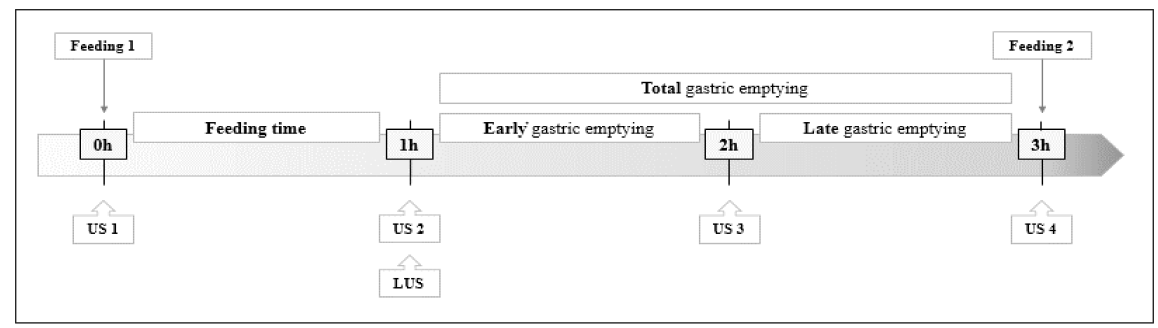

FIGURE 1

\section{Hosted file}

TABLE 1 for PP SUBMISSION 11-24-2020.pdf available at https://authorea.com/users/378999/ articles/495373-ultrasound-assessment-of-gastric-emptying-in-premature-infants-treatedwith-non-invasive-ventilatory-support

\section{Hosted file}


TABLE 2 for PP SUBMISSION 11-24-2020.pdf available at https://authorea.com/users/378999/ articles/495373-ultrasound-assessment-of-gastric-emptying-in-premature-infants-treatedwith-non-invasive-ventilatory-support

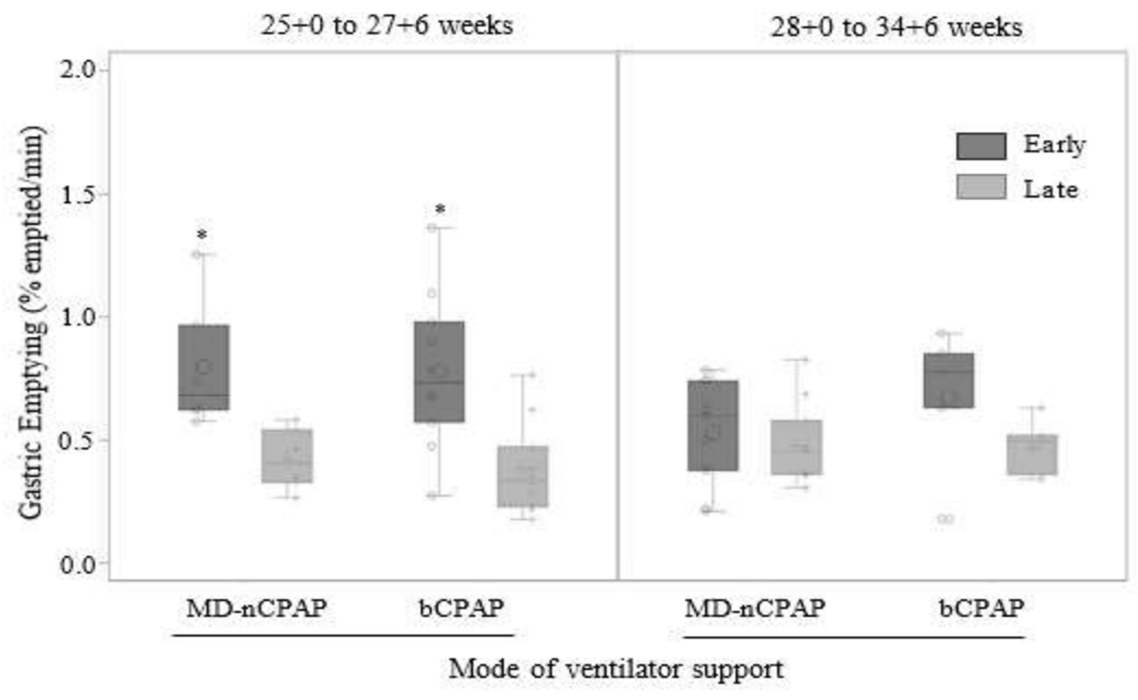

FIGURE 2a

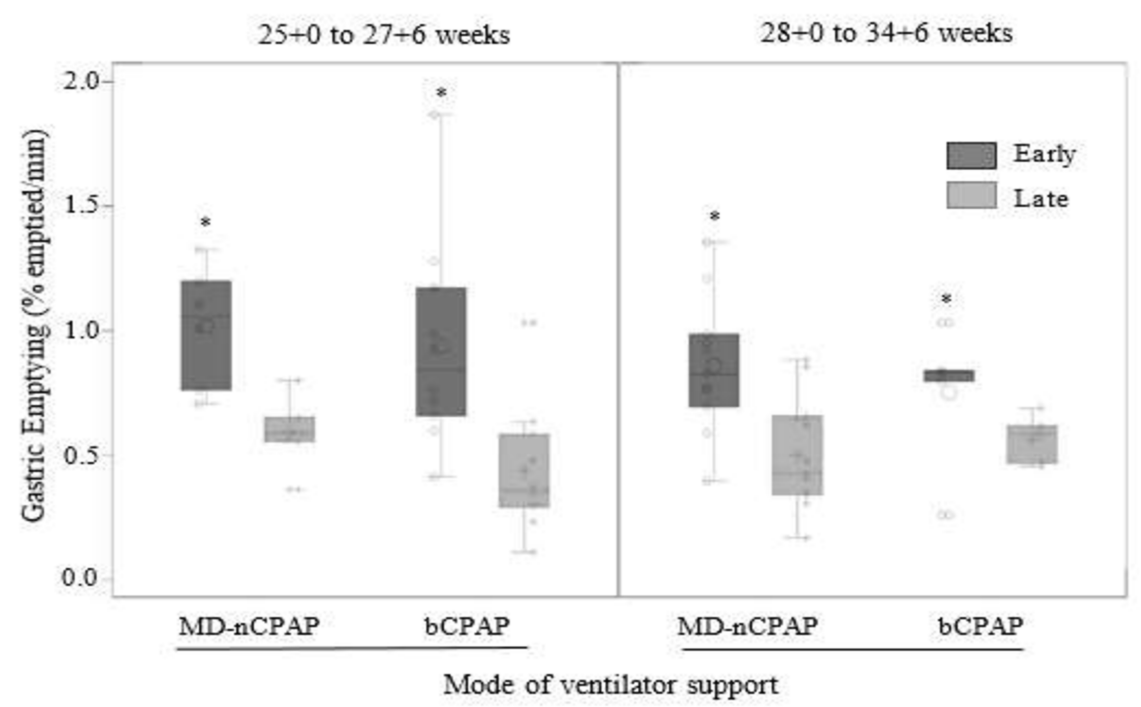

FIGURE 2b 


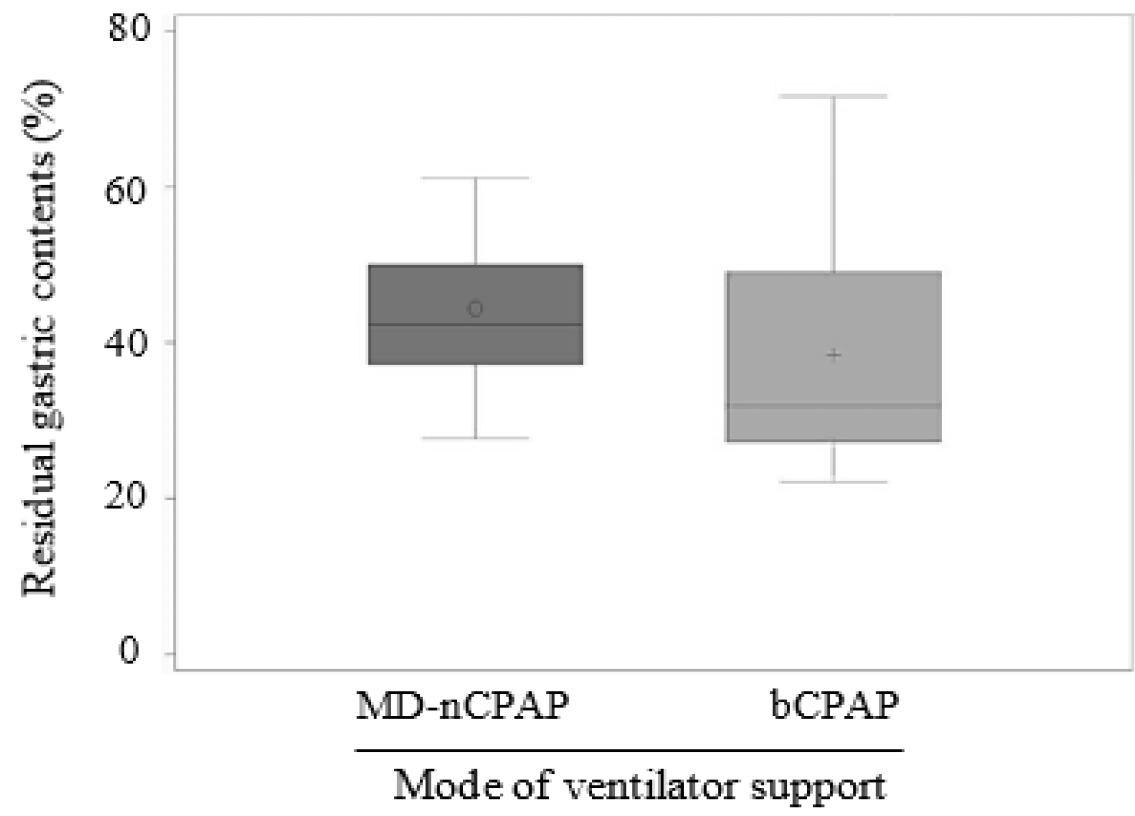

FIGURE 3a

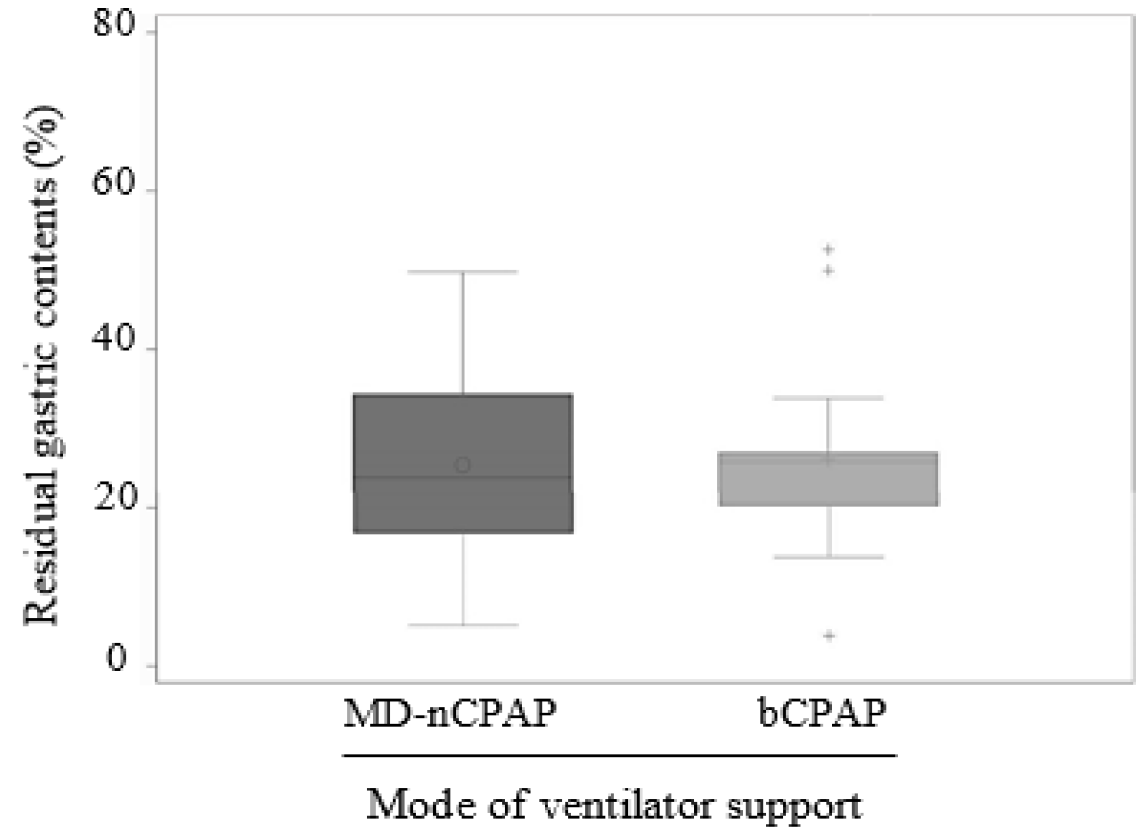

\section{FIGURE 3b}

